\title{
Severe desaturations increase psychomotor vigilance task-based median reaction time and number of lapses in obstructive sleep apnoea patients
}

\author{
Samu Kainulainen $\mathbb{1}^{1,2}$, Brett Duce $\mathbb{1}^{3,4}$, Henri Korkalainen (101,2, \\ Arie Oksenberg $\mathbb{1}^{5}$, Akseli Leino (10 $\mathbb{1}^{1,2}$, Erna S. Arnardottir $\mathbb{1}^{6,7}$, Antti Kulkas $\mathbb{1}^{1,8}$, \\ Sami Myllymaa (10 ${ }^{1,2}$, Juha Töyräs (10 ${ }^{1,2,9}$ and Timo Leppänen (101,2 \\ Affiliations: ${ }^{1}$ Dept of Applied Physics, University of Eastern Finland, Kuopio, Finland. ${ }^{2}$ Diagnostic Imaging \\ Center, Kuopio University Hospital, Kuopio, Finland. ${ }^{3}$ Sleep Disorders Centre, Dept of Respiratory and Sleep \\ Medicine, Princess Alexandra Hospital, Brisbane, Australia. ${ }^{4}$ Institute for Health and Biomedical Innovation, \\ Queensland University of Technology, Brisbane, Australia. ${ }^{5}$ Sleep Disorders Unit, Loewenstein Hospital - \\ Rehabilitation Center, Raanana, Israel. ${ }^{6}$ Dept of Engineering, Reykjavik University, Reykjavik, Iceland. ${ }^{7}$ Internal \\ Medicine Services, Landspitali - The National University Hospital of Iceland, Reykjavik, Iceland. ${ }^{8}$ Dept of \\ Clinical Neurophysiology, Seinäjoki Central Hospital, Seinäjoki, Finland. ${ }^{9}$ School of Information Technology \\ and Electrical Engineering, The University of Queensland, Brisbane, Australia.
}

Correspondence: Samu Kainulainen, Dept of Applied Physics, University of Eastern Finland, P.O. Box 1627 (Canthia), 70211 Kuopio, Finland. E-mail: samu.kainulainenduef.fi

@ERSpublications

Parameters considering characteristic properties of desaturations have a significant association with impaired vigilance, highlighting the importance of developing methods beyond the AHI for a more detailed assessment of OSA severity http://bit.ly/2veqxD9

Cite this article as: Kainulainen S, Duce B, Korkalainen $\mathrm{H}$, et al. Severe desaturations increase psychomotor vigilance task-based median reaction time and number of lapses in obstructive sleep apnoea patients. Eur Respir J 2020; 55: 1901849 [https://doi.org/10.1183/13993003.01849-2019].

ABSTRACT Current diagnostic parameters estimating obstructive sleep apnoea (OSA) severity have a poor connection to the psychomotor vigilance of OSA patients. Thus, we aimed to investigate how the severity of apnoeas, hypopnoeas and intermittent hypoxaemia is associated with impaired vigilance.

We retrospectively examined type I polysomnography data and corresponding psychomotor vigilance tasks (PVTs) of 743 consecutive OSA patients (apnoea-hypopnoea index (AHI) $\geqslant 5$ events $\cdot \mathrm{h}^{-1}$ ). Conventional diagnostic parameters (e.g. AHI and oxygen desaturation index (ODI)) and novel parameters (e.g. desaturation severity and obstruction severity) incorporating duration of apnoeas and hypopnoeas as well as depth and duration of desaturations were assessed. Patients were grouped into quartiles based on PVT outcome variables. The odds of belonging to the worst-performing quartile were assessed. Analyses were performed for all PVT outcome variables using binomial logistic regression.

A relative $10 \%$ increase in median depth of desaturations elevated the odds $\left(\mathrm{OR}_{\text {range }} 1.20-1.37, \mathrm{p}<0.05\right)$ of prolonged mean and median reaction times as well as increased lapse count. Similarly, an increase in desaturation severity $\left(\mathrm{OR}_{\text {range }} 1.26-1.52, \mathrm{p}<0.05\right)$ associated with prolonged median reaction time. Female sex $\left(\mathrm{OR}_{\text {range }} 2.21-6.02, \mathrm{p}<0.01\right)$, Epworth Sleepiness Scale score $\left(\mathrm{OR}_{\text {range }} 1.05-1.07, \mathrm{p}<0.01\right)$ and older age $\left(\mathrm{OR}_{\text {range }} 1.01-1.05, \mathrm{p}<0.05\right)$ were significant risk factors in all analyses. In contrast, increases in conventional AHI, ODI and arousal index were not associated with deteriorated PVT performance.

These results show that our novel parameters describing the severity of intermittent hypoxaemia are significantly associated with increased risk of impaired PVT performance, whereas conventional OSA severity and sleep fragmentation metrics are not. These results underline the importance of developing the assessment of OSA severity beyond the AHI.

Copyright $\odot$ ERS 2020. This article is open access and distributed under the terms of the Creative Commons Attribution Non-Commercial Licence 4.0. 


\section{Introduction}

Inadequate sleep, due to sleep disorders and shortened sleep times, is widely recognised as a significant public health burden in Western countries [1]. Good quality sleep is crucial for maintaining neurocognitive performance [1]. Conversely, an increasing number of occupational accidents and absences, as well as traffic accidents, are caused by factors decreasing sleep quality [1]. In Australia alone, the estimated cost of inadequate sleep was USD 45 billion in healthcare and society during 2016-2017 [1]. Obstructive sleep apnoea (OSA) is one of the most prevalent sleep disorders, affecting $\geqslant 20 \%$ of the adult population in Western countries [2-4]. OSA is attributed to daytime symptoms of shortened daytime sleep latencies as well as chronic fatigue and sleepiness [5-7]. Furthermore, OSA is related to poor neurocognitive performance and inability to sustain attention [8-10].

Neurocognitive disorders, defined in the Diagnostic and Statistical Manual of Mental Disorders, cover the domains of learning and memory, language, executive functioning and complex attention, among others [11]. Psychomotor vigilance task (PVT) evaluates the domain of complex attention, by measuring repeated responses to visual stimuli to assess the subject's ability to sustain attention [8, 12]. PVT is a fast, objective and easily interpretable test that has very little learning effect [13]. PVT is considered highly reliable and sensitive to the effect of sleep deprivation on cognitive performance and demonstrates clearly the cumulative worsening of performance during the task when an individual is unable to sustain attention [14]. As clinical resources for objective evaluation of sleepiness are limited and subjective questionnaires unreliable, PVT could be used, for example, as an initial assessment for the fitness to drive [15], with the constraint that driving also involves other complex skills in addition to sustained attention.

Studies have found impairments in PVT performance with acute sleep deprivation [16] and chronic sleep restriction [9], but also high inter-individual differences in responses [17]. Furthermore, the association between worsening PVT outcomes and the usual clinical parameters that describe the severity of OSA (i.e. the apnoea-hypopnoea index (AHI)) or degree of sleep fragmentation (i.e. arousal index) is equivocal [9, 18-22]. Previous studies have shown that an increase in AHI is not directly linked to impaired vigilance $[9,22]$. Even though patients suffering from severe OSA have on average inferior PVT performance compared to healthy individuals, no significant differences in PVT performance between OSA severity groups have been reported $[9,22,23]$. However, this lack of differences in OSA patients could be related to arbitrary, outdated and suboptimal severity classification thresholds for the AHI [24, 25].

Even though the AHI does not correlate well with reduced PVT performance, PACK et al. [9] reported that impaired performance in PVT seems to result from long-term sleep deprivation and insufficient sleep durations in OSA patients. Furthermore, previous studies suggest that the level of nocturnal intermittent hypoxaemia negatively affects PVT performance in OSA patients [10, 18]. SFORZA et al. [18] showed that the number of lapses correlated moderately with the minimum and mean blood oxygen saturation ( $\mathrm{r}=-0.454$ and -0.421 , respectively), but this was achieved using an atypically long reaction time limit of $1000 \mathrm{~ms}$. In addition, we recently demonstrated that longer and deeper desaturations, regardless of the AHI, significantly increases the risk of objectively measured excessive daytime sleepiness [7].

Based on this apparent connection between intermittent hypoxaemia and impaired daytime functioning of OSA patients, we hypothesise that novel, more sophisticated diagnostic parameters that consider the duration of respiratory events as well as the depth and duration of desaturations have a stronger association to impaired PVT performance than conventional severity indices such as AHI and oxygen desaturation index (ODI). In this study, we investigate which OSA severity indices are associated with poor PVT performance in a large patient cohort. In addition, we examine how the severity of desaturations affects PVT performance independently of the number and total duration of the respiratory events.

\section{Patients and methods}

This was a retrospective analysis of 912 consecutive patients undergoing polysomnography (PSG) in the Sleep Disorders Center of Princess Alexandra Hospital (Brisbane, Australia) due to the suspicion of OSA. The institutional human research ethics committee of the Princess Alexandra Hospital approved the retrospective data collection (HREC/16/QPAH/021, LNR/2019/QMS/54313). Patients who failed to complete PVTs $(n=32)$, had AHI $<5$ events $\cdot h^{-1}(n=134)$ or had missing demographic information $(n=3)$ were excluded. Thus, the studied patient population consisted of 743 individuals, who had successfully undergone type 1 diagnostic PSG, had AHI $\geqslant 5$ events $\cdot h^{-1}$ upon PSG and successfully completed the PVT. The PVT was conducted between 19:00 and 21:00 h prior to the PSG. The demographic information of

This article has supplementary material available from erj.ersjournals.com

Received: 18 Sept 2019 | Accepted after revision: 17 Jan 2020 
the whole patient population is presented in table 1. PSG recordings were conducted and scored using the Compumedics Grael acquisition system and Compumedics ProFusion PSG 4 software (Compumedics, Abbotsford, Australia) during 2015-2017.

All PSG recordings were scored manually by experienced sleep technicians, who regularly participate in scoring concordance activities. All apnoeas, hypopnoeas and desaturations were scored manually in conformity with the America Academy of Sleep Medicine 2012 guidelines [26]. A hypopnoea was scored if $a \geqslant 30 \%$ reduction in nasal pressure amplitude was observed for $\geqslant 10 \mathrm{~s}$ and was associated with either $\geqslant 3 \%$ desaturation and/or an arousal (recommended hypopnoea rule 4A [26]). An apnoea was scored if a $\geqslant 90 \%$ reduction in amplitude for $\geqslant 10 \mathrm{~s}$ in the thermistor signal was observed. An oxygen desaturation event was manually scored if a $\geqslant 3 \%$ drop in oxygen saturation signal $\left(S_{\mathrm{pO}_{2}}\right)$ measured by pulse oximeter, was observed. Desaturation events were scored from the last sampling point before the onset of the desaturation to the point when recovery reached the baseline (figure 1). If the baseline was not reached, the end-point was determined at the start of plateau after the desaturation (figure 1).

For PVT, the Psychology Experiment Building Language PVT programme on an ASUS transformer pad (Taipei, Taiwan) with an attached keyboard was used [27]. PVT was performed using the 10-min protocol, with 120 visual stimuli occurring at 2-10-s intervals. Patients were instructed to monitor the tablet display and press a response button using the index finger or thumb on their dominant hand as soon as the pink stimulus appeared on the screen. Standard PVT outcomes were recorded: median reaction time (RT), mean reciprocal reaction time (RRT), mean of the slowest 10\% RT, mean of the fastest 10\% RT and the number of lapses (RT >500 ms).

Raw $S_{\mathrm{pO}_{2}}$ signals, hypnograms and the information (e.g. duration, start and end points) of scored apnoea, hypopnoea, arousal and desaturation events were exported from ProFusion to MatLab (version R2018b; MathWorks, Natick, MA, USA). Total sleep time (TST) and percentages of non-rapid eye movement sleep and rapid eye movement sleep from TST were based on manual sleep staging results. AHI, oxygen desaturation index (ODI), arousal index, average nocturnal oxygen saturation (mean $S_{\mathrm{pO}_{2}}$ ), minimum nocturnal oxygen saturation (min $S_{\mathrm{pO}_{2}}$ ), time spent with $S_{\mathrm{pO}_{2}}<90 \%\left(\mathrm{t}_{90 \%}\right)$ and the mean and median depth of desaturations were computed. In addition, novel parameters (obstruction duration, desaturation duration, desaturation severity and obstruction severity) (table 2) described in our previous studies [28-30], were computed based on the respiratory and desaturation event information and raw $S_{\mathrm{pO}_{2}}$ signal with custom-made MatLab functions. All types of apnoeas (obstructive, mixed, central) were considered in the analyses and none of the patients had central sleep apnoea, i.e. percentage of central events $<50 \%$ of all events. Physiologically irrelevant $S_{\mathrm{pO}_{2}}$ values $(\leqslant 50 \%)$ within desaturations or in minimum $S_{\mathrm{pO}_{2}}$ were considered as artefacts and were not used in analyses.

Obstruction duration parameter represents the percentage of the total duration of apnoeas (figure 1 and table 2) and hypopnoeas (figure 1 and table 2) from TST. Similarly, the desaturation duration parameter

\begin{tabular}{|c|c|}
\hline Patients n (male \%) & 743 (58.7) \\
\hline Depression & 140 (18.8) \\
\hline Hypertension & 329 (44.3) \\
\hline Smoker & $117(15.7)$ \\
\hline COPD & $82(11.0)$ \\
\hline Age years & $56.8(46.4-66.7)$ \\
\hline $\mathrm{BMI} \mathrm{kg} \cdot \mathrm{m}^{-2}$ & $35.1(30.3-41.3)$ \\
\hline TST h & $5.0(4.2-5.9)$ \\
\hline NREM \% & $83.3(78.3-88.4)$ \\
\hline REM \% & $16.6(11.6-21.7)$ \\
\hline AHI events. $\mathbf{h}^{-1}$ & $23.7(12.5-45.0)$ \\
\hline ESS score & $10(6-14)$ \\
\hline RRT $1 / \mathrm{ms}$ & $2.6(2.2-2.9)$ \\
\hline Median RT ms & $380.0(340.0-445.0)$ \\
\hline Fastest $10 \% \mathrm{RT}$ ms & $297.0(277.0-335.8)$ \\
\hline Slowest $10 \%$ RT ms & $691.0(544.3-1081.0)$ \\
\hline PVT lapses ${ }^{\#} n$ & $13(5-36)$ \\
\hline
\end{tabular}

Data are presented as $\mathrm{n}(\%)$ or median (interquartile range), unless otherwise stated. COPD: chronic obstructive pulmonary disease; BMI: body mass index; TST: total sleep time; NREM: non-rapid eye movement sleep; REM: rapid eye movement sleep; AHI: apnoea-hypopnoea index; ESS: Epworth Sleepiness Scale; RRT: mean reciprocal reaction time; RT: reaction time. ${ }^{\#}$ : reaction times $>500 \mathrm{~ms}$. 

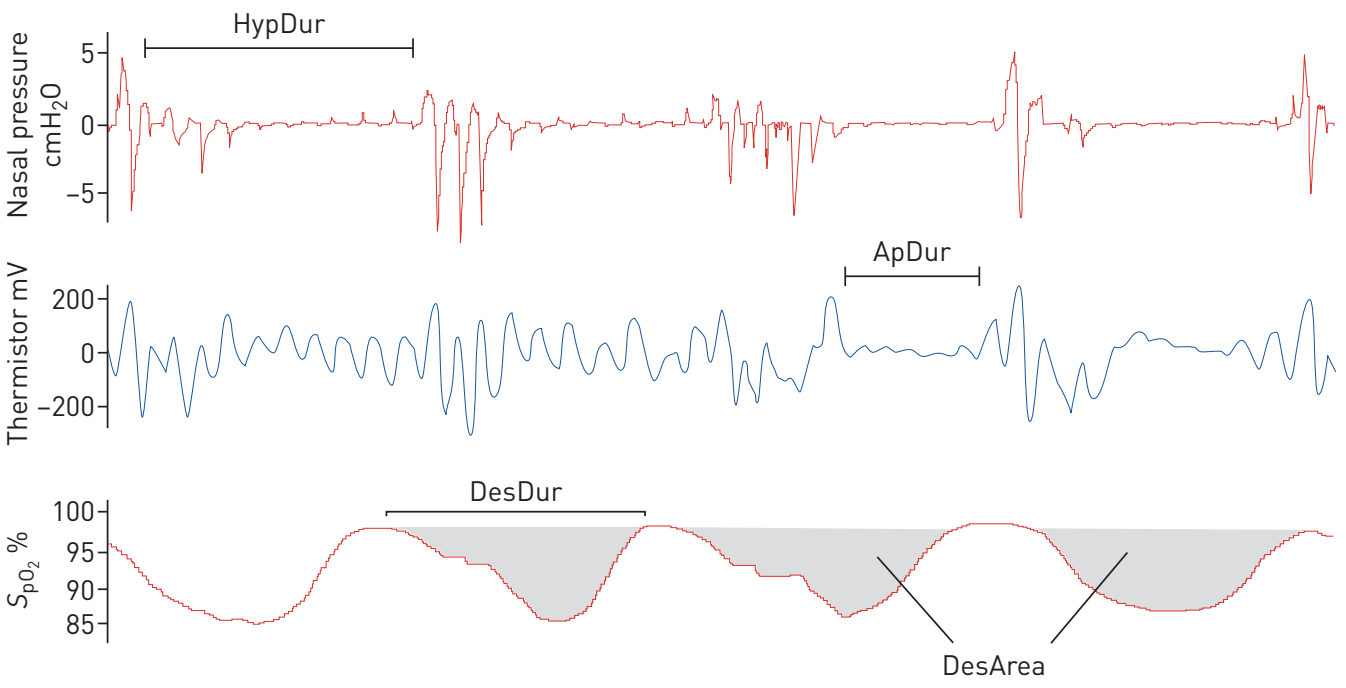

FIGURE 1 Schematic representation of the variables related to novel parameters. HypDur: duration of hypopnoea(s); ApDur: duration of apnoea (s); DesDur: duration of desaturation(s); DesArea: area of a desaturation(s).

represents the percentage of the total duration of desaturations (figure 1 and table 2) from TST. Desaturation severity parameter represents the severity of desaturation events (i.e. desaturation area) (figure 1 and table 2) normalised by TST. Desaturation areas were computed by using the start and end time of each individual desaturation event and numerically integrating the corresponding desaturation area (figure 1). In addition, the obstruction severity parameter is defined as a TST normalised sum of multiplications between apnoea or hypopnoea duration, and corresponding desaturation area (table 2).

For statistical analyses, patients were grouped into PVT performance quartiles (Q1-Q4) based on each of the five PVT outcome variables (tables 1 and 3). After grouping, multivariate binomial logistic regression was used to investigate which parameters were associated with impaired PVT performance. Multivariate binomial logistic regression was chosen due to the assumption that the connection between OSA severity indices and poor PVT performance is nonlinear and multivariate, and because the method does not require any transformations for the PVT outcome variables. The patients belonging to the worst-performing quartiles were compared to the patients belonging to the best-performing quartiles. Models were first adjusted for sex, age, body mass index (BMI), Epworth Sleepiness Scale (ESS) score, smoking status and existing comorbidities (depression, hypertension and COPD). The coexistence of

\section{TABLE 2 Equations for computation of the conventional and novel parameters described}

\begin{tabular}{|c|c|}
\hline $\mathrm{AHI}$ events: $\mathrm{h}^{-1}$ & $\frac{\mathrm{L}+\mathrm{K}}{\mathrm{TST}_{\mathrm{h}}}$ \\
\hline ODI events $\cdot \mathrm{h}^{-1}$ & $\frac{\mathrm{P}}{\mathrm{TST}_{\mathrm{h}}}$ \\
\hline Obstruction duration \% & $\frac{\sum_{\mathrm{n}=1}^{\mathrm{L}} \operatorname{ApDur}_{\mathrm{n}}+\sum_{\mathrm{m}=1}^{\mathrm{K}} \operatorname{HypDur}_{\mathrm{m}}}{\text { TST }} \times 100 \%$ \\
\hline Desaturation duration \% & $\frac{\sum_{\mathrm{n}=1}^{\mathrm{P}} \operatorname{DesDur}_{\mathrm{n}}}{\mathrm{TST}} \times 100 \%$ \\
\hline Desaturation severity \% & $\frac{\sum_{\mathrm{n}=1}^{\mathrm{P}} \operatorname{DesArea}_{\mathrm{n}}}{\text { TST }}$ \\
\hline Obstruction severity s\% & $\frac{\sum_{m=1}^{K}\left(\text { HypDur }_{m} \times \text { DesArea }_{m}\right)+\sum_{n=1}^{L}\left(\text { ApDur }_{n} \times \text { DesArea }_{n}\right)}{\text { TST }}$ \\
\hline
\end{tabular}

AHI: apnoea-hypopnoea index; ODI: oxygen desaturation index; L: number of apnoeas; K: number of hypopnoeas; $\mathrm{TST}_{\mathrm{h}}$ : total sleep time computed in hours from polysomnography (PSG); P: number of desaturations; ApDur: duration of apnoea(s); HypDur: duration of hypopnoea(s); TST: total sleep time computed in seconds from polysomnography; DesDur: duration of desaturation(s); DesArea: area of desaturation (s\%). 
TABLE 3 Demographic and polysomnographic data in the worst-performing psychomotor vigilance task (PVT) quartile (Q4) and best-performing quartile (Q1) based on each examined PVT outcome

\begin{tabular}{|c|c|c|c|c|c|c|c|c|c|c|}
\hline & \multicolumn{2}{|c|}{ RRT $1 / \mathrm{ms}$} & \multicolumn{2}{|c|}{ Median RT ms } & \multicolumn{2}{|c|}{ Slowest $10 \%$ RT ms } & \multicolumn{2}{|c|}{ Fastest $10 \% \mathrm{RT}$ ms } & \multicolumn{2}{|c|}{ Lapses $\#$} \\
\hline & Q1 (>2.9) & Q4 $(<2.2)$ & $01(<340.0)$ & Q4 $(>445.0)$ & Q1 $(<544.3)$ & Q4 (>1081.0) & Q1 $(<277.0)$ & Q4 (>335.8) & Q1 (<5) & Q4 $(>36)$ \\
\hline Patients (female \%) & $154(21.4)$ & $202(58.4)$ & $189(22.2)$ & 184 (59.8) & $186(26.8)$ & $186(50.0)$ & $192(27.1)$ & $186(59.7)$ & $189(25.9)$ & $180(56.1)$ \\
\hline Depression & $21(13.6)$ & $46(22.8)$ & 25 (13.2) & $41(22.3)$ & $26(14.0)$ & 40 (21.5) & $33(17.2)$ & $38(20.4)$ & 26 (13.8) & $43(23.9)$ \\
\hline Hypertension & 50 (32.5) & 92 (45.5) & 69 (36.5) & 85 (46.2) & $69(37.1)$ & $90(48.4)$ & 70 (36.5) & $86(46.2)$ & 65 (34.4) & $83(46.1)$ \\
\hline Smoker & $26(16.9)$ & 36 (17.8) & 33 (17.5) & 34 (18.5) & 27 (14.5) & $36(19.4)$ & 33 (17.2) & $31(16.7)$ & $31(16.4)$ & 34 (18.9) \\
\hline COPD & $12(7.8)$ & $25(12.4)$ & $14(7.4)$ & 22 (11.2) & 12 (6.5) & 24 (12.9) & 17 (8.9) & 18 (9.7) & $14(7.4)$ & 23 (12.8) \\
\hline Age years & $52.5(42.8-63.7)$ & $58.4(48.9-68.1)$ & $54.1(42.9-65.4)$ & $58.9(49.9-69.4)$ & $52.5(42.8-63.7)$ & $58.7(49.0-69.0)$ & $52.5(43.0-64.8)$ & $58.4(50.2-68.1)$ & $51.3(42.8-62.1)$ & $58.9(49.4-68.5)$ \\
\hline BMI $\mathrm{kg} \cdot \mathrm{m}^{-2}$ & $34.3(30.1-40.4)$ & 35.0 (30.1-41.9) & $34.0(30.1-40.4)$ & $35.0(30.1-42.0)$ & 34.9 (30.1-41.8) & $35.0(29.8-40.7)$ & 35.4 (30.1-40.5) & $35.4(30.4-41.6)$ & $33.9(29.2-40.4)$ & $34.9(30.2-42.1)$ \\
\hline TST h & $5.0(4.0-5.9)$ & $5.0(4.0-5.9)$ & $5.0(4.0-5.9)$ & $5.0(4.0-6.0)$ & $5.1(4.2-6.0)$ & $5.0(3.9-5.9)$ & $5.1(4.0-5.9)$ & $4.9(4.0-5.8)$ & $5.2(4.3-5.9)$ & $4.9(3.9-5.9)$ \\
\hline NREM \% & $82.0(77.5-87.2)$ & $82.9(78.3-88.2)$ & $82.0(77.6-87.6)$ & 82.9 (78.3-88.3) & $82.1(77.4-88.5)$ & $82.6(78.0-88.2)$ & 82.5 (78.4-88.5) & $82.6(78.3-87.8)$ & $82.1(77.7-88.2)$ & $82.5(78.1-88.2)$ \\
\hline REM \% & $18.1(12.8-22.5)$ & $17.2(11.8-21.7)$ & $18.0(12.4-22.4)$ & $17.1(11.7-21.7)$ & $18.0(11.5-22.6)$ & $17.4(11.8-22.0)$ & $17.5(11.6-21.7)$ & $17.4(12.2-21.7)$ & $17.9(11.8-22.3)$ & $17.5(11.8-21.9)$ \\
\hline AHI events $\cdot h^{-1}$ & $25.8(13.0-47.2)$ & $22.2(11.5-42.1)$ & $27.0(13.5-45.7)$ & $22.5[11.8-42.1)$ & $27.5(13.0-49.4)$ & $21(11.4-42.3)$ & $24.7(12.0-43.7)$ & $22.8(11.5-42.1)$ & $27.5(12.9-48.7)$ & $22.9(11.8-46.9)$ \\
\hline ESS score & $9(5-13)$ & $11(6-16)$ & $9(5-13)$ & $11(6-16)$ & $9(5-13)$ & $11(6-17)$ & $10(6-14)$ & $11(5-16)$ & $9(5-12)$ & $11(6-17)$ \\
\hline
\end{tabular}

Data are presented as $\mathrm{n}$ (\% of population) or median (interquartile range). RRT: mean reciprocal reaction time; RT: reaction time; BMI: body mass index; TST: total sleep time; NREM: non-rapid eye movement sleep; REM: rapid eye movement sleep; AHI: apnoea-hypopnoea index; ESS: Epworth Sleepiness Scale. ${ }^{\#}$ : reaction times $>500 \mathrm{~ms}$.

depression and all the other comorbidities were reported based on medical history or interview in the sleep clinics. The partial maximum likelihood estimates of $\beta$-coefficients for belonging to the worst-performing PVT quartile were computed individually for every investigated parameter and adjusting factor. Second, the model was adjusted for TST, AHI, arousal index and obstruction duration to examine how the severity of desaturations affects PVT performance independently. This additionally adjusted model was used for two reasons. First, to test the hypoxaemia hypothesis in a more detailed manner by controlling the number and duration of respiratory events. Second, we wanted to avoid the use of predefined limits for OSA severity classes, and rather treat AHI as a continuous variable. After computing the $\beta$-coefficients for all models, the coefficients were converted to odds ratios according to the logit-function. As examined parameters differ substantially in the order of magnitude, their values were normalised by the maximum value of each parameter in question before the analyses. However, the normalised $1 \%$ increase, e.g. in AHI would have produced an increase of 1.7 events $\cdot \mathrm{h}^{-1}$ and for median desaturation depth an increase of $0.29 \%$. Thus, the parameter values were further scaled to equal a $10 \%$ relative change to achieve a comparable and clinically more meaningful increase.

\section{Results \\ Comparison between the worst- and best-performing PVT quartiles}

The comparison between Q1 and Q4 was performed individually for every examined OSA severity metric with two different multivariate logistic regression models. When the model was adjusted for demographics, subjective sleepiness and comorbidities, an increase in either $t_{90 \%}$, desaturation severity parameter, mean desaturation depth or median desaturation depth significantly elevated the odds of longer median RT (OR 1.17-1.26). Slower RRT (OR 1.24-1.26) (table 4) was significantly associated with an increase in mean and median desaturation depth. Only an increase in $t_{90 \%}(\mathrm{OR} 1.18, \mathrm{p}=0.04)$ and median desaturation depth (OR 1.20, $\mathrm{p}=0.02$ ) significantly elevated the odds of having a higher number of lapses. An increase in desaturation severity parameter $(\mathrm{p}=0.06)$ and mean desaturation depth $(\mathrm{p}=0.09)$ were at the borderline of significantly elevating the odds of belonging to Q4 based on lapses. In addition, an increase in the median desaturation depth showed significantly (OR 1.25, $\mathrm{p}=0.04$ ) elevated odds of belonging to Q4 based on the fastest $10 \% \mathrm{RT}$.

With the model additionally adjusted for TST, arousal index, AHI and obstruction duration parameter, the worsening in all examined hypoxaemia parameters significantly elevated the odds of belonging to Q4 based on the number of lapses; however, a decrease in the mean $S_{\mathrm{pO}_{2}}(\mathrm{p}=0.10)$ and increase in mean desaturation depth $(p=0.12)$ did not significantly affect the odds of having longer median RT compared to other hypoxaemia parameters (table 4 ). An increase either in desaturation severity parameter ( $\mathrm{OR} 1.43, \mathrm{p}=0.01$ ) or median desaturation depth (OR 1.36, $\mathrm{p}=0.02$ ) significantly elevated the odds of belonging to Q4 based on RRT. In addition, investigation of slowest 10\% RT quartiles showed that an increase in obstruction severity parameter (OR 1.39, $\mathrm{p}=0.04)$, $\mathrm{t}_{90 \%}(\mathrm{OR} 1.19, \mathrm{p}=0.04)$ or median depth (OR 1.27, $\mathrm{p}=0.02$ ) elevated the odds of belonging to Q4. None of the parameters were significantly associated with the odds of belonging to Q4 based on the fastest 10\% RT. Results of similar analyses comparing Q4 to all other quartiles are presented in supplementary table S1. 
TABLE 4 The odds of belonging to the worst-performing psychomotor vigilance task (PVT) quartile (Q4) compared to best-performing quartile (Q1) when parameter values are increased by $10 \%$

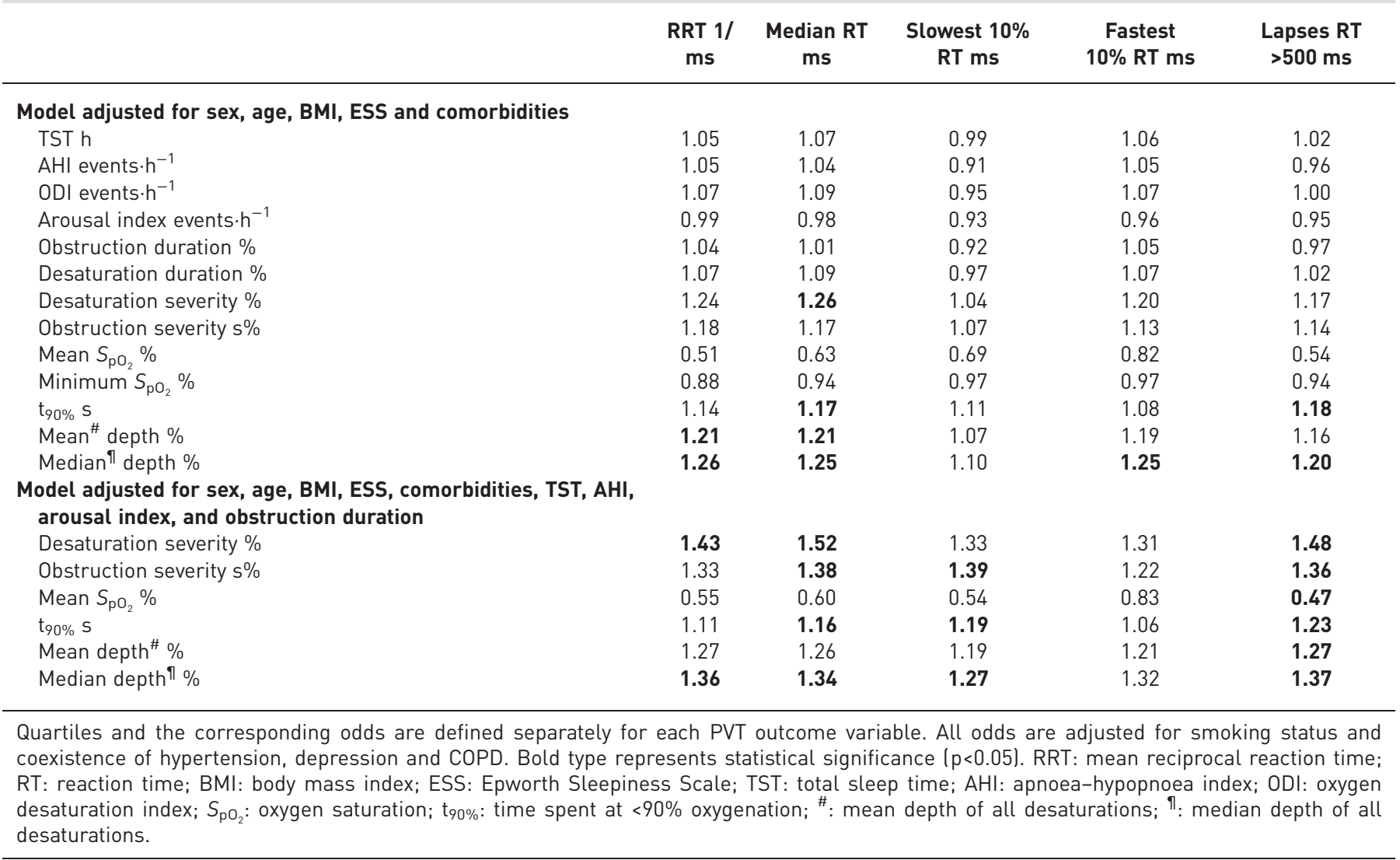

\section{Demographic variables as risk factors for impaired PVT performance}

When demographic risk factors, subjective sleepiness (ESS) and risk of comorbidities were assessed, only female sex, higher ESS score and older age were significantly associated with elevated odds of impaired PVT performance. Female sex $\left(\mathrm{OR}_{\text {range }} 2.21-6.02, \mathrm{p}<0.01\right)$ and increase in $\mathrm{ESS}\left(\mathrm{OR}_{\text {range }} 1.05-1.07, \mathrm{p}<0.01\right)$ were significant risk factors with all PVT outcome variables, whereas increasing age was significant in all analyses with quartiles based on RRT, median RT and lapses $\left(\mathrm{OR}_{\text {range }} 1.01-1.05, \mathrm{p}<0.05\right)$. BMI, smoking and coexistence of depression, COPD or hypertension had no association with impaired PVT performance.

\section{Discussion}

In this study, we investigated the role of conventional and novel PSG parameters in predicting PVT performance in a sample of 743 OSA patients. We found that our novel parameters describing the severity of intermittent hypoxaemia are significantly associated with increased risk of impaired PVT performance, whereas conventional OSA severity and sleep fragmentation metrics (i.e. AHI, ODI and arousal index) are not. This finding is supported by our previous study [7], showing that OSA-related objective daytime sleepiness is more strongly associated with the severity of individual desaturations than the number of apnoeas and hypopnoeas. Furthermore, the present results correspond with previous studies [8, 9, 18-22], by showing that conventional AHI-based assessments of OSA have little connection to poor PVT performance. These findings highlight, together with recent studies [7, 24, 29, 31], the clinical importance of a more detailed analysis of PSG recordings when assessing OSA severity, symptoms and daytime functioning. As the presented novel parameters are based on routine manual scoring of PSG, they can be easily implemented by clinics without any extra work required of technical or medical staff [32].

Based on our results, the severity of nocturnal intermittent hypoxaemia is the strongest PSG-related indicator of poor PVT performance. The more severe desaturations leading to longer median reaction times and a higher number of lapses were consistent within each subgroup analysis (table 4) and consistent with previous studies $[10,18]$. Our results show that larger desaturation areas and deeper desaturations negatively affect the vigilance and the ability to sustain attention in OSA patients. Interestingly, the duration of desaturations was not a significant factor in elevating the odds of impaired 
PVT performance (table 4). It has been shown that OSA-related intermittent hypoxaemia causes neuronal brain damage and similar changes as in ischaemic injury [33]. Furthermore, a recent study shows that systemic intermittent hypoxaemia readily translates to cerebral hypoxaemia in healthy individuals, indicating that there are limits to which the autoregulation system can prevent hypoxaemia in the cerebral vasculature [34]. The combination of our results with these findings suggests that the depth of desaturations, in conjunction with the repetitive failure of the cerebral autoregulation system, may drive the cognitive impairment seen in OSA patients.

The highest and most significant odds ratios were induced by desaturation severity parameter, median desaturation depth and mean desaturation depth across all analyses. Comparisons between the demographically adjusted and additionally adjusted models (table 4) show that when the number and duration of the apnoeas and hypopnoeas are invariant, increasing depth and area of desaturations will elevate the odds of prolonged reaction times. It has been previously shown that patients with similar AHI values can exhibit highly different responses in peripheral blood oxygenation [29]. These findings suggest that there exist substantial differences between patients in how rapidly peripheral oxygenation decreases and how fast reoxygenation to baseline occurs. Therefore, further studies are warranted to consider the morphology of desaturations, as well as the decrease and recovery rates of $S_{\mathrm{pO}_{2}}$ within desaturation events, and the association of these factors with cardiovascular outcomes and daytime symptoms in OSA patients.

As we hypothesised, the more detailed diagnostic parameters have a significantly stronger connection to impaired PVT performance than AHI or other conventional PSG parameters. Against this hypothesis, our results indicate that the duration of apnoeas, hypopnoeas and desaturations does not seem to affect PVT performance as much as the depth of desaturations. The obstruction severity parameter is defined as the multiplications of desaturation areas and durations of the corresponding respiratory event, whereas desaturation severity is independent of respiratory event duration. Therefore, it is reasonable that the obstruction severity parameter has less significance than the desaturation severity parameter, as the duration of the events was found to be insignificant. It is noteworthy that obstruction severity was developed for home sleep apnoea testing and therefore, no arousal-related hypopnoeas could have been considered. This is persuasive for the development of PSG-modified obstruction severity parameter, where severity of arousals could be quantified using, e.g. the frequency content and the duration of arousal in a similar manner compared to the desaturation area. Based on the results achieved for the detailed hypoxaemia parameters with both adjustment schemes, it can be speculated that deteriorated vigilance is much more dependent on physiological consequences caused by apnoeas and hypopnoeas rather than the number or the duration of events per se. In addition, as the emphasis of this study was to assess the role of intermittent hypoxaemia in vigilance failure, only a limited number of electroencephalogram (EEG) and sleep fragmentation metrics were investigated in this study. Therefore, further studies are warranted for detailed EEG analyses and their connection to the impaired PVT performance to gain more comprehensive information on the factors causing vigilance deterioration.

This study was based on a relatively large, well-balanced clinical sample of patients whose demographics, symptoms and comorbidities reflect well general OSA populations (table 1), making the obtained results generalisable to clinical populations. However, clinical and epidemiological populations can include other comorbid sleep disorders, such as insomnia and restless legs syndrome among others, which may have a confusing effect on findings related to PVT outcomes. In addition, the present results show that female sex, subjective sleepiness and older age together with OSA are significant predictors of poor PVT performance. Similar findings have also been reported in other studies investigating cognitive impairment in OSA patients and healthy individuals $[9,19,20,22,35]$. Moreover, in our recent studies, we reported that a larger desaturation area is a significant predictor of objective daytime sleepiness and the most detrimental health consequences of OSA $[7,29,31]$. Together with these findings, this study highlights the urgent need to develop the diagnostics of OSA beyond the AHI. Diagnosis should be made more comprehensive by characterising OSA severity with demographical information and with more detailed parameters that are better related to the symptoms, daytime functioning and, for example, cardiovascular comorbidities of OSA. To support the idea of utilising these new metrics in normal clinical workflow, we have already published a plug-in for RemLogic (Natus Medical, Middleton, WI, USA) that computes these new metrics automatically based on routine manual scoring of the sleep recording [32]. These metrics could also be implemented in other clinical software in a similar manner.

This study has certain limitations. First, the PVT outcome variables used in this study were the standard statistical parameters from the series of 120 reaction times. Individuals have natural differences in their repeated reaction times, and therefore the PVT analysis could benefit from utilising the complete time series. Nevertheless, the standard parameters used in this study enables us to compare with the existing literature regarding the connection of OSA to PVT outcomes. Second, patients completed their PVT prior to PSG. Individuals suffering from OSA tend to have shorter TST in PSG than normally [36], which can 
affect the odds obtained for TST. It is acknowledged that TST modulates the values of AHI and ODI to a great extent; however, all novel parameters are also normalised by TST. Furthermore, in a recent study, it was reported that severities of individual apnoeas, hypopnoeas and desaturations increase towards morning [37]. Therefore, it is reasonable to assume that mean and minimum $S_{\mathrm{pO}_{2}}$ would decrease, and $t_{90 \%}$ as well as the mean and median depth of desaturations, would increase alongside increasing TST. Thus, we believe that the timing of the PVT does not diminish the obtained results. Third, although the models were adjusted with comorbidities, a complete record of patients' medication at the time of PSG and PVT was not available. Psychoactive and sedative medications can especially affect the reaction times and thus PVT performance, which could affect the computed odds ratios to some extent. Fourth, this study did not involve a control group of healthy individuals. However, the objective of this study was to investigate which are the most significant PSG parameters associated with impaired PVT performance in OSA patients. Therefore, patients without OSA were excluded due to the assumption that their impaired vigilance is not caused by the same factors compared to those suffering from OSA. Fifth, no data on patient's habitual sleep (e.g. questionnaire, sleep diary or long-term actigraphy) were available. Short sleep duration and chronic sleep deprivation are significantly associated with sleepiness and can, therefore, affect neurocognitive performance [38]; thus, the lack of this information is a limitation of the present study.

In conclusion, we have shown that parameters quantifying desaturations based on their characteristic properties have a significant association with impaired vigilance and ability to sustain attention. Furthermore, an increase in AHI or ODI does not significantly elevate the odds of having impaired PVT performance. These results highlight the importance of developing methods for a more detailed assessment of OSA severity and comprehensive analysis of PSGs. This would enhance the assessment of OSA severity and improve the estimation of risk and severity of related daytime symptoms.

Author contributions: J. Töyräs, A. Kulkas, S. Myllymaa, A. Oksenberg and T. Leppänen devised the project and the main conceptual ideas for the analysis. S. Kainulainen, H. Korkalainen and B. Duce carried out the data collection and preparation. S. Kainulainen, H. Korkalainen, A. Leino, B. Duce and E.S. Arnardottir carried out the analyses and interpretation. S. Kainulainen drafted the manuscript and prepared the figures. All the authors have revised the manuscript critically, approved the version submitted for publication and have agreed to be accountable for all aspects of the work.

Conflict of interest: S. Kainulainen reports grants from Academy of Finland (grant number 313697), the Research Committee of the Kuopio University Hospital Catchment Area (project numbers 5041779 and 5041768), the Competitive State Research Financing of Expert Responsibility Area of Tampere University Hospital (grants VTR3221, VTR3228 and EVO2089), Päivikki and Sakari Sohlberg Foundation and the Research Foundation of the Pulmonary Diseases, during the conduct of the study. B. Duce has nothing to disclose. H. Korkalainen reports grants from Academy of Finland (grant number 313697), the Research Committee of the Kuopio University Hospital Catchment Area (project numbers 5041780 and 5041767), Respiratory Foundation of Kuopio Region, Päivikki and Sakari Sohlberg Foundation, the Research Foundation of the Pulmonary Diseases, Foundation of the Finnish Anti-Tuberculosis Association, during the conduct of the study. A. Oksenberg has nothing to disclose. A. Leino reports grants from the Research Committee of the Kuopio University Hospital Catchment Area (project number 5041776), Päivikki and Sakari Sohlberg Foundation, the Research Foundation of the Pulmonary Diseases, the Finnish Cultural Foundation and Respiratory Foundation of Kuopio Region, during the conduct of the study. E.S. Arnardottir reports grants, personal fees and non-financial support from Nox Medical, personal fees from Philips and ResMed, outside the submitted work. A. Kulkas reports grants from Seinäjoki Central Hospital, Competitive State Research Financing of Expert Responsibility Area of Tampere University Hospital (VTR3221 and VTR3228 and Tampere Tuberculosis foundation, during the conduct of the study. S. Myllymaa reports grants from Academy of Finland (grant number 313697), the Research Committee of the Kuopio University Hospital Catchment Area (project numbers 5041770), Paulo Foundation and Tampere Tuberculosis Foundation, during the conduct of the study. J. Töyräs reports grants from Academy of Finland (decision number 313697), Kuopio University Hospital (project number 5041767) and Business Finland (decision number 5133/31/2018), during the conduct of the study. T. Leppänen reports grants from the Research Committee of the Kuopio University Hospital Catchment Area for the State Research Funding (project number 5041767), Academy of Finland (decision numbers 313697 and 323536), Tampere Tuberculosis Foundation and Respiratory Foundation of Kuopio Region, during the conduct of the study.

Support statement: During conduction of the present study, funding was received from the Research Committee of the Kuopio University Hospital Catchment Area for the State Research Funding (projects 5041767, 5041768, 5041770, 5041776, 5041779 and 5041780), the Academy of Finland (decision numbers 313697 and 323536), Seinäjoki Central Hospital, the Competitive State Research Financing of Expert Responsibility Area of Tampere University Hospital (grants VTR3221 and VTR3228), Business Finland (decision number 5133/31/2018), Paulo Foundation, Päivikki and Sakari Sohlberg Foundation, The Research Foundation of the Pulmonary Diseases, Finnish Cultural Foundation, Tampere Tuberculosis Foundation, Finnish Anti-Tuberculosis Foundation and the Respiratory Foundation of Kuopio Region. Funding information for this article has been deposited with the Crossref Funder Registry.

\section{References}

Hillman D, Mitchell S, Streatfeild J, et al. The economic cost of inadequate sleep. Sleep 2018; 41: zsy083. Dempsey JA, Veasey SC, Morgan BJ, et al. Pathophysiology of sleep apnea. Physiol Rev 2010; 90: 47-112. Heinzer R, Vat S, Marques-Vidal P, et al. Prevalence of sleep-disordered breathing in the general population: the HypnoLaus study. Lancet Respir Med 2015; 3: 310-318. 
4 Senaratna C, Perret J, Lodge C, et al. Prevalence of obstructive sleep apnea in the general population: a systematic review. Sleep Med Rev 2017; 34: 70-81.

5 Garbarino S, Scoditti E, Lanteri P, et al. Obstructive sleep apnea with or without excessive daytime sleepiness: clinical and experimental data-driven phenotyping. Front Neurol 2018; 9: 505.

6 Oksenberg A, Arons E, Nasser K, et al. Severe obstructive sleep apnea: sleepy versus nonsleepy patients. Laryngoscope 2010; 120: 643-648.

7 Kainulainen S, Töyräs J, Oksenberg A, et al. Severity of desaturations reflects OSA-related daytime sleepiness better than AHI. J Clin Sleep Med 2019; 15: 1135-1142.

8 Arnardottir ES, Bjornsdottir E, Olafsdottir KA, et al. Obstructive sleep apnoea in the general population: highly prevalent but minimal symptoms. Eur Respir J 2016; 47: 194-202.

9 Pack AI, Maislin G, Staley B, et al. Impaired performance in commercial drivers: role of sleep apnea and short sleep duration. Am J Respir Crit Care Med 2006; 174: 446-454.

10 Tanno S, Tanigawa T, Maruyama $\mathrm{K}$, et al. Sleep-related intermittent hypoxia is associated with decreased psychomotor vigilance in Japanese community residents. Sleep Med 2017; 29: 7-12.

11 American Psychiatric Association (APA). Diagnostic and Statistical Manual of Mental Disorders. 5th Edn. APA, Washington, 2013.

12 Dinges DF, Powell JW. Microcomputer analyses of performance on a portable, simple visual RT task during sustained operations. Behav Res Methods Instrum Comput 1985; 17: 652-655.

13 Basner M, Hermosillo E, Nasrini J, et al. Repeated administration effects on psychomotor vigilance test performance. Sleep 2018; 41: zsx187.

14 Lim J, Dinges DF. Sleep deprivation and vigilant attention. Ann NY Acad Sci 2008; 1129: 305-322.

15 Jackson ML, Croft RJ, Kennedy GA, et al. Cognitive components of simulated driving performance: sleep loss effects and predictors. Accid Anal Prev 2013; 50: 438-444.

16 Goel N, Basner M, Dinges DF. Phenotyping of neurobehavioral vulnerability to circadian phase during sleep loss. Meth Enzymol 2015; 552: 285-308.

17 Van Dongen HPA, Baynard MD, Maislin G, et al. Systematic interindividual differences in neurobehavioral impairment from sleep loss: evidence of trait-like differential vulnerability. Sleep 2004; 27: 423-433.

18 Sforza E, Haba-Rubio J, De Bilbao F, et al. Performance vigilance task and sleepiness in patients with sleep-disordered breathing. Eur Respir J 2004; 24: 279-285.

19 Parekh A, Mullins AE, Kam K, et al. Slow-wave activity surrounding stage N2 K-complexes and daytime function measured by psychomotor vigilance test in obstructive sleep apnea. Sleep 2019; 42: zsy256.

20 Li Y, Vgontzas A, Kritikou I, et al. Psychomotor vigilance test and its association with daytime sleepiness and inflammation in sleep apnea: clinical implications. J Clin Sleep Med 2017; 13: 1049-1056.

21 Kim H, Dinges DF, Young T. Sleep-disordered breathing and psychomotor vigilance in a community-based sample. Sleep 2007; 30: 1309-1316.

22 Batool-Anwar S, Kales SN, Patel SR, et al. Obstructive sleep apnea and psychomotor vigilance task performance. Nat Sci Sleep 2014; 6: 65-71.

23 Wong KKH, Marshall NS, Grunstein RR, et al. Comparing the neurocognitive effects of $40 \mathrm{~h}$ sustained wakefulness in patients with untreated OSA and healthy controls. J Sleep Res 2008; 17: 322-330.

24 Korkalainen H, Töyräs J, Nikkonen S, et al. Mortality-risk-based apnea-hypopnea index thresholds for diagnostics of obstructive sleep apnea. J Sleep Res 2019; 28: e12855.

25 Shahar E. Apnea-hypopnea index: time to wake up. Nat Sci Sleep 2014; 6: 51-56.

26 Berry RB, Budhiraja R, Gottlieb DJ, et al. Rules for scoring respiratory events in sleep: update of the 2007 AASM Manual for the Scoring of Sleep and Associated Events. J Clin Sleep Med 2012; 8: 597-619.

27 Mueller ST, Piper BJ. The Psychology Experiment Building Language (PEBL) and PEBL test battery. J Neurosci Methods 2014; 222: 250-259.

28 Kulkas A, Tiihonen P, Eskola K, et al. Novel parameters for evaluating severity of sleep disordered breathing and for supporting diagnosis of sleep apnea-hypopnea syndrome. J Med Eng Technol 2013; 37: 135-143.

29 Kulkas A, Tiihonen P, Julkunen P, et al. Novel parameters indicate significant differences in severity of obstructive sleep apnea with patients having similar apnea-hypopnea index. Med Biol Eng Comput 2013; 51: 697-708.

30 Muraja-Murro A, Nurkkala J, Tiihonen P, et al. Total duration of apnea and hypopnea events and average desaturation show significant variation in patients with a similar apnea-hypopnea index. J Med Eng Technol 2012; 36: 393-398.

31 Muraja-Murro A, Kulkas A, Hiltunen M, et al. Adjustment of apnea-hypopnea index with severity of obstruction events enhances detection of sleep apnea patients with the highest risk of severe health consequences. Sleep Breath 2014; 18: 641-647.

32 Leppänen T, Särkkä M, Kulkas A, et al. RemLogic plug-in enables clinical application of apnea-hypopnea index adjusted for severity of individual obstruction events. J Med Eng Technol 2016; 40: 119-126.

33 Gagnon K, Baril A, Cary A, et al. Mild cognitive impairment in obstructive sleep apnea. Sleep Med 2013; 14: el32.

34 Rupp T, Peyrard A, Tamisier R, et al. Cerebral and muscle oxygenation during intermittent hypoxia exposure in healthy humans. Sleep 2016; 39: 1197-1199.

35 Blatter K, Graw P, Münch M, et al. Gender and age differences in psychomotor vigilance performance under differential sleep pressure conditions. Behav Brain Res 2006; 168: 312-317.

36 Byun J, Kim KT, Moon $\mathrm{H}$, et al. The first night effect during polysomnography, and patients' estimates of sleep quality. Psychiatry Res 2019; 274: 27-29.

37 Nikkonen S, Töyräs J, Mervaala E, et al. Intra-night variation in apnea-hypopnea index affects diagnostics and prognostics of obstructive sleep apnea. Sleep Breath 2019; in press [https://doi.org/10.1007/s11325-019-01885-5].

38 Prasad B, Steffen AD, Van Dongen HPA, et al. Determinants of sleepiness in obstructive sleep apnea. Sleep 2018; 41: zsx199. 